\title{
EXPLORING THE INTRABINARY SHOCK FROM THE REDBACK MILLISECOND PULSAR PSR J2129-0429
}

\author{
C. Y. Hui ${ }^{1}$, C. P. $\mathrm{Hu}^{2}$, S. M. PARK ${ }^{1}$, J. TAKATA ${ }^{3}$, K. L. $\mathrm{LI}^{4}$, P. H. T. TAM ${ }^{5}$, L. C. C. $\mathrm{LIN}^{6}$, \\ A. K. H. Kong ${ }^{4}$, K. S. Cheng ${ }^{3}$, AND CHUngleE $\mathrm{Kim}^{7}$ \\ ${ }^{1}$ Department of Astronomy and Space Science, Chungnam National University, Daejeon, Korea; cyhui@cnu.ac.kr \\ ${ }^{2}$ Graduate Institute of Astronomy, National Central University, Jhongli 32001, Taiwan \\ ${ }^{3}$ Department of Physics, University of Hong Kong, Pokfulam Road, Hong Kong \\ ${ }^{4}$ Institute of Astronomy and Department of Physics, National Tsing Hua University, Hsinchu, Taiwan \\ 5 Institute of Astronomy and Space Science, Sun Yat-Sen University, Guangzhou 510275, China \\ ${ }^{6}$ Institute of Astronomy and Astrophysics, Academia Sinica, Taiwan \\ ${ }^{7}$ Department of Physics and Astronomy, Seoul National University, Korea \\ Received 2015 January 2; accepted 2015 February 11; published 2015 March 11
}

\begin{abstract}
We have investigated the intrabinary shock emission from the redback millisecond pulsar PSR J2129-0429 with XMM-Newton and Fermi. Orbital modulation in X-ray and UV can be clearly seen. The X-ray modulation of this pulsar has a double-peaked structure with a dip in between. The observed X-rays are non-thermal dominant and can be modeled by a power-law with $\Gamma \sim 1.2$. An intrabinary shock may have been the origin of the observed $X-$ rays with the UV light curve resulting from the ellipsoidal modulation of the companion. Modeling the UV light curve requires a large viewing angle. The heating effect of the UV light curve is found to be negligible which suggests the high energy radiation beam of PSR J2129-0429 is not directed toward its companion. On the other hand, no significant orbital modulation can be found in $\gamma$-rays which suggests the majority of the $\gamma$-rays come from the pulsar.
\end{abstract}

Key words: gamma rays: stars - pulsars: individual (PSR J2129-0429) - X-rays: binaries

\section{INTRODUCTION}

A new population of eclipsing millisecond pulsars (MSPs) has emerged in the last $5 \mathrm{yr}$, which is characterized by an orbital period $P_{b} \lesssim 20 \mathrm{hr}$ and a companion mass $M_{c} \sim 0.1-0.5$ $M_{\odot}$. These MSPs are commonly referred to as "redbacks" (Roberts 2013; Hui 2014). Currently, 18 redback MSPs have been discovered. ${ }^{8}$ Through coordinated multiwavelength searches, the population of redbacks is growing (Kong et al.2012, 2014; Hui et al. 2015). These MSPs play a crucial role in exploring the transition between a rotation-powered system and an accretion-powered system.

To better understand this transition, it is important to probe the interactions between MSPs and their companions. In a rotation-powered state, the collision between the pulsar wind and the mass outflow from the companion can produce an intrabinary shock. Non-thermal X-rays from the accelerated particles in the shock region can be modulated by the orbital period; this phenomenon has been observed in various redbacks/black widows (e.g., Bogdanov et al. 2005, 2011, 2014; Tam et al. 2010; Archibald et al. 2010; Huang et al. 2012; Hui et al. 2014; Gentile et al. 2014).

PSR J2129-0429 is one of the poorly studied redbacks. It was discovered in the radio pulsation search of the $\gamma$-ray source 2FGL J2129.8-0428 with the Green Bank Telescope (Hessels et al. 2011; Ray et al. 2012). Its dispersion measure suggests a distance of $d \sim 0.9 \mathrm{kpc}$ (Ray et al. 2012; Roberts 2014). ${ }^{9}$ Its spin period and orbital period are $P_{s} \sim 7.62 \mathrm{~ms}$ and $P_{b} \sim 0.64$ day, respectively (cf. Table 2 in Ray et al. 2012). Roberts (2014) reported a surface dipolar magnetic field of $B \sim 1.6 \times 10^{9} \mathrm{G}$ for this pulsar, which implies a characteristic

\footnotetext{
8 See http://apatruno.wordpress.com/about/millisecond-pulsar-catalogue/ for updated information.

9 http://fermi.gsfc.nasa.gov/science/mtgs/symposia/2014/program/ 10A_Roberts.pdf
}

age and spin-down luminosity of the order of $\tau \sim 4 \times 10^{8} \mathrm{yr}$ and $\dot{E} \sim 3 \times 10^{34} \mathrm{erg} \mathrm{s}^{-1}$ respectively. These values suggest PSR J2129-0429 is a young energetic MSP with a relatively high surface magnetic field (Roberts 2014).

The minimum mass of its companion has been found to be $>0.37 M_{\odot}$ using radio timing (Ray et al. 2012). Optical observations suggest its companion is significantly bloated with a Roche lobe filling factor of $\sim 95 \%$ (Bellm et al. 2013). The X-ray position of PSR J2129-0429 has recently been constrained by using archival Swift/XRT data (i.e., source J2129B in Linares 2014). Using the data obtained by XMMNewton, Roberts (2014) has found significant X-ray orbital modulation from this system. However, the detailed emission properties of PSR J2129-0429 have not yet been reported. In this Letter, we report the results from our investigation of PSR J2129-0429 by using the X-ray and UV data obtained by $X M M-N e w t o n$. We also present the analysis of the $\gamma$-ray data obtained by the Fermi Gamma-ray Space Telescope.

\section{XMM-NEWTON OBSERVATIONS AND DATA ANALYSIS}

The XMM-Newton observations of PSR J2129-0429 started on 2013 October 28 with a total exposure of $\sim 80 \mathrm{ks}$ (ObsID: 0725070101; PI: Roberts). Using XMM Science Analysis Software (XMMSAS version 13.5.0), we reduced and filtered the data using standard procedure. The effective exposures for MOS1, MOS2, and PN after filtering are found to be $\sim 79.1 \mathrm{ks,}$ $\sim 79.0 \mathrm{ks}$, and $\sim 77.5 \mathrm{ks}$, respectively. This X-ray observation covers $\sim 1.4$ orbital cycles of PSR J2129-0429. All the EPIC data are found to be unaffected by CCD pile-up.

With all the EPIC data merged, we produced an X-ray image of the $3^{\prime} \times 3^{\prime}$ field around PSR J2129-0429 (see Figure 1). With the aid of the XMMSAS task edetect_chain, the X-ray position of PSR J2129-0429 determined by this observation is 
R.A. $=21^{\mathrm{h}} 29^{\mathrm{m}} 45^{\mathrm{s}} .250$, decl. $=-04^{\circ} 29^{\prime} 07^{\prime} \cdot 95(\mathrm{~J} 2000)$ with a statistical uncertainty of $\sim 0{ }^{\prime \prime} 1{ }^{10}$ This is consistent with the $\mathrm{X}$ ray source J2129B reported by Linares (2014) but with the position better constrained.

In Figure 1, we note that the X-ray image of PSR J21290429 is slightly extended toward the west. Such a feature can also be seen in the images from individual cameras. In MOS1/2 data, it is detected as a distinct source at R.A. $=21^{\mathrm{h}} 29^{\mathrm{m}} 43^{\mathrm{s}} .749$ decl. $=-04^{\circ} 29^{\prime} 07^{\prime \prime} .83(\mathrm{~J} 2000)$ with a statistical error of $\sim 0.7$. We do not find any identified object within $5^{\prime \prime}$ from this position in SIMBAD and NED. Also, we do not find any optical/IR counterpart in the USNO-B1.0 and 2MASS point source catalogs. Within $8^{\prime \prime}$ around its nominal position, we have collected $\sim 230$ net counts from all cameras. We found that its spectra can be described by an absorbed power-law (PL) model with $N_{\mathrm{H}}<5 \times 10^{20} \mathrm{~cm}^{-2}$ and a photon index of $\Gamma \sim 1.6$. Its absorption-corrected flux in $0.3-10 \mathrm{keV}$ is $f_{x} \sim 1.5 \times 10^{-14} \mathrm{erg} \mathrm{cm}^{-2} \mathrm{~s}^{-1}$. Given the moderate angular resolution of XMM-Newton, we cannot unambiguously determine whether this feature is indeed a distinct source or a bowshock nebula associated with PSR J2129-0429. Since the nature of this feature is uncertain, we excluded its contribution in all subsequent analyses.

Before any temporal analysis was performed, we applied a barycentric correction to the arrival times of all the events by using the updated planetary ephemeris JPL DE405. We extracted the events from a circular source region of $15^{\prime \prime}$ radius centered at the X-ray position of PSR J2129-0429 so as to exclude the aforementioned feature in the west. After subtracting the background by sampling the events from the source-free regions in individual cameras, there are 718, 732, and 2382 counts extracted from MOS1, MOS2, and PN CCDs, respectively. To improve the photon statistic, we merged all the EPIC data and folded the background-subtracted X-ray light curve at the orbital period of PSR J2129-0429, which is shown in the top panel of Figure 2. Since the radio timing model of PSR J2129-0429 is not publicly available, we arbitrarily chose

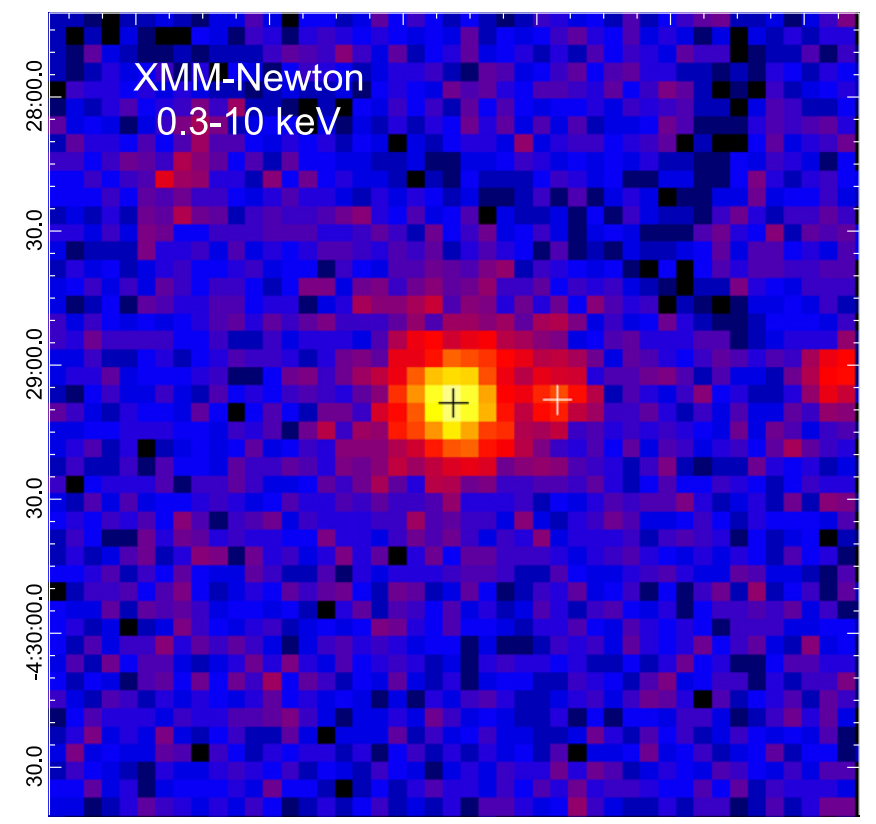

Figure 1. X-ray image of $3^{\prime} \times 3^{\prime}$ field around PSR J2129-0429 as observed by XMM-Newton with all EPIC data merged. The black and white crosses illustrate the positions of the pulsar and a nearby feature, respectively. the day that this observation started (i.e., MJD 56593) to be phase zero throughout our investigation.

The maxima of the X-ray modulation are found at the orbital phases $\phi \sim 0.1$ and $\phi \sim 0.45$. In between these two maxima, there is a dip at $\phi \sim 0.25$. We have inspected the backgroundsubtracted light curves from individual cameras and found the dip at $\phi \sim 0.25$ in all three cameras. Therefore, we confirmed that this feature is genuine. We further examined the X-ray orbital modulation of PSR J2129-0429 by dividing the EPIC data into soft band (0.3-2 keV) and hard band (2-10 keV) and investigated how the X-ray hardness ratio, which is defined as (hard-soft)/(hard+soft), varies across the orbit. We found that the X-ray hardness ratio decreases in $\phi \sim 0.45-0.7$ (see the middle panel in Figure 2) which is apparently in unison with the decrement of X-ray intensity (top panel of Figure 2). On the other hand, at phase $\phi \sim 0.25$ where a dip has been observed, there is an indication of the enhancement of X-ray hardness.

The optical monitor (OM) on board XMM-Newton has also observed PSR J2129-0429 in fast mode with the UVW1 filter $(291 \mathrm{~nm})$ for a total integration time of $\sim 75 \mathrm{ks}$. PSR J21290429 is the only source detected in this OM observation. This provides us with temporal and photometric information of PSR J2129-0429 in the UV regime. The background-subtracted UV light curve of PSR J2129-0429 was automatically extracted by the XMMSAS tool omfchain. After a barycentric correction, we folded the UV light curve at the orbital period of PSR J2129-0429 with phase zero defined at the same epoch as for the X-ray light curve. The resultant UV light curve is shown in the bottom panel of Figure 2. Two UV peaks with a phase separation of $\sim 0.5$ are observed in one orbit. The minima at phases $\sim 0.25$ and $\sim 0.75$ apparently coincide with the dip and the minimum of the X-ray orbital modulation. To convert the magnitude into energy flux, we adopted a scale that the flux of Vega corresponds to $0.025 \mathrm{mag}$ in UVW1 filter. The energy flux changes from $\sim 5.5 \times 10^{-17}$ to $\sim 1.0 \times 10^{-16}$ erg cm $\mathrm{cm}^{-2} \mathrm{~s}^{-1} \AA^{-1}$ across the orbit.

To investigate its X-ray spectral properties, we extracted the source and background spectra from the same regions adopted in the temporal analysis. The spectra obtained from all three cameras are fitted simultaneously to the tested models. All the uncertainties quoted in this paper are $1 \sigma$ for two parameters of interest (i.e., $\Delta \chi^{2}=2.3$ ).

We found that a simple absorbed PL model can describe its phase-averaged X-ray spectra reasonably well $\left(\chi^{2}=59.98\right.$ for 59 degrees of freedom (dof)). The best-fit model yields a column density of $N_{\mathrm{H}}<6 \times 10^{19} \mathrm{~cm}^{-2}$, a photon index of $\Gamma$ $=1.25 \pm 0.04$, and a normalization of $(1.84 \pm 0.06) \times 10^{-5}$ photons $\mathrm{keV}^{-1} \mathrm{~cm}^{-2} \mathrm{~s}^{-1}$ at $1 \mathrm{keV}$. To investigate whether the phase-averaged spectrum requires an additional thermal component for modeling, we added a blackbody (BB) on top of the best-fit PL model. It results in a goodness-of-fit with $\chi^{2}=54.45$ for 57 dof which indicates that the additional BB component is required at a confidence level of $\sim 94 \%$. The PL + BB fit yields $N_{\mathrm{H}}<3.9 \times 10^{20} \mathrm{~cm}^{-2}, \Gamma=1.15_{-0.04}^{+0.09}$, a PL model normalization of $1.66_{-0.19}^{+0.27} \times 10^{-5}$ photons $\mathrm{keV}^{-1} \mathrm{~cm}^{-2} \mathrm{~s}^{-1}$ at $1 \mathrm{keV}$, a blackbody temperature of $k T=0.16_{-0.08}^{+0.06} \mathrm{keV}$ with an emission radius of $R=92.9_{-49.3}^{+121.3} d_{1 \mathrm{kpc}} \mathrm{m}$, where $d_{1 \mathrm{kpc}}$ is the distance to the pulsar in units of $1 \mathrm{kpc}$. The best-fit PL+BB model and the observed spectra are shown in Figure 3. The unabsorbed energy flux in

$\overline{10 \text { Absolute astrometric accuracy of EPIC is }} \sim 1$."2. 


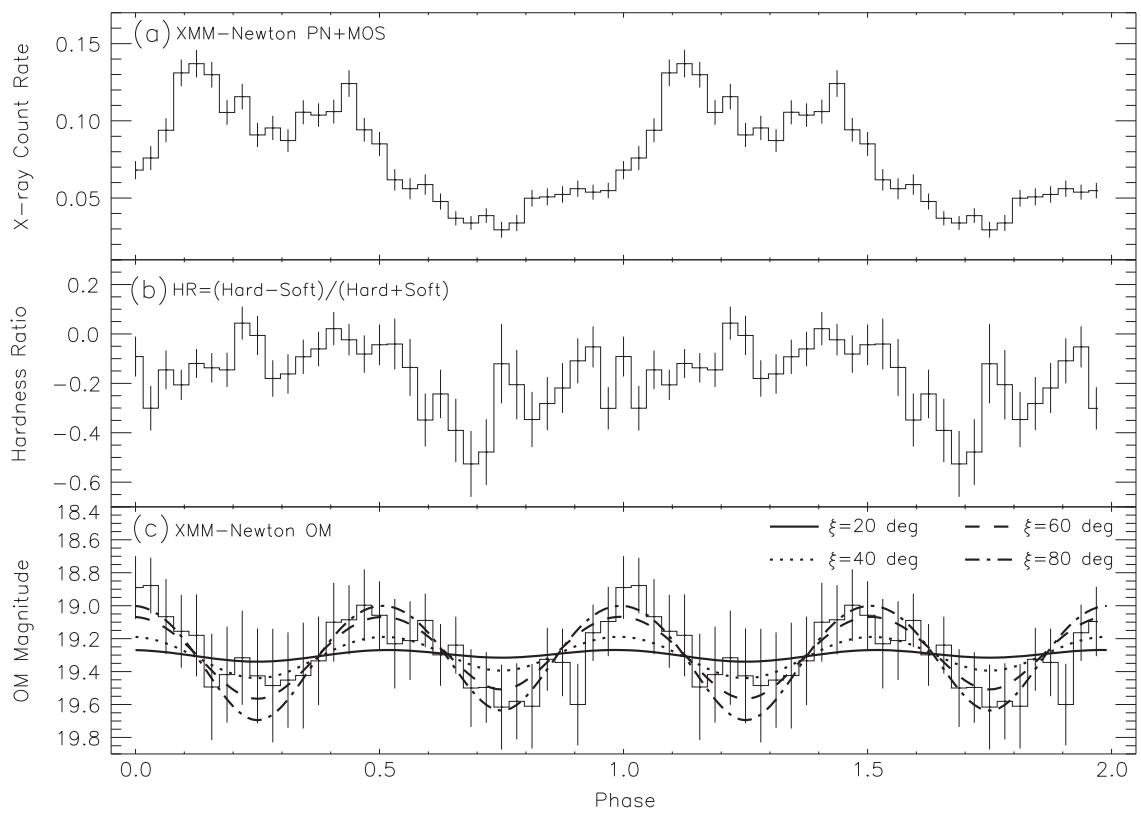

Figure 2. Background-subtracted light curve of PSR J2129-0429 as observed by XMM-Newton in 0.3-10 keV with the data from all EPIC cameras combined (upper panel), the X-ray hardness variation (middle panel), and the UV light curve as observed by XMM-Newton OM with the model light curves overlaid (lower panel; see Section 4 for details). The orbital period adopted for folding is 0.64 day as reported by Ray et al. (2012). The epoch of phase zero is set at MJD 56593 . Two periods of orbital motion are shown for clarity.

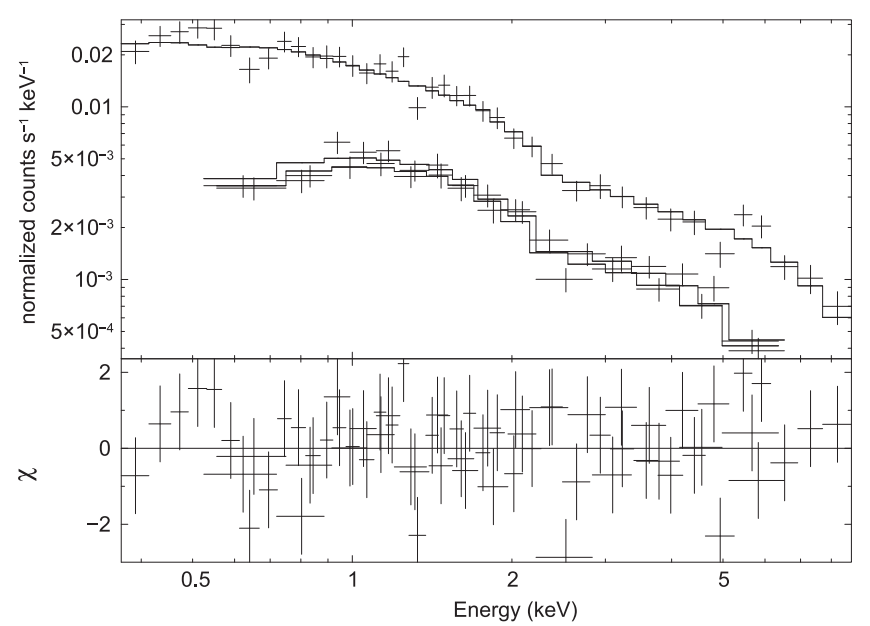

Figure 3. Phase-averaged X-ray spectra of PSR J2129-0429 as observed by $X M M-N e w t o n$ PN (upper spectrum) and MOS1/2 cameras (lower spectra) and simultaneously fitted to an absorbed power-law plus blackbody model (upper panel) and contribution to the $\chi$ statistic (lower panel).

$0.3-10 \mathrm{keV}$ is $f_{x} \sim 2.1 \times 10^{-13} \mathrm{erg} \mathrm{cm}^{-2} \mathrm{~s}^{-1}$. The $\mathrm{BB}$ component contributes $\sim 2 \%$ of the total flux in this band.

Motivated by the variation of X-ray hardness across the orbit (cf. Figure 2), we performed a phase-resolved analysis to investigate how the emission nature varies with orbital phase. We divided the orbit into two intervals, $\phi=0.0-0.5$ and $\phi=0.5-1$, which encompass the peak and the trough of the orbital modulation respectively. For $\phi=0.0-0.5$, we found that a single PL model is already sufficient for modeling the observed spectrum $\left(\chi^{2}=57.57\right.$ for 58 dof $)$ which yields $N_{\mathrm{H}}=5.2_{-5.2}^{+28.1} \times 10^{19} \mathrm{~cm}^{-2}, \Gamma=1.10 \pm 0.06$ and a PL model normalization of $2.9_{-0.2}^{+0.3} \times 10^{-5}$ photons $\mathrm{keV}^{-1} \mathrm{~cm}^{-2} \mathrm{~s}^{-1}$ at $1 \mathrm{keV}$. Adding a $\mathrm{BB}$ component does not result in any improvement $\left(\chi^{2}=57.51\right.$ for 56 dof $)$. The best-fit PL+BB model in this phase interval yields $N_{\mathrm{H}}=2.9_{-2.9}^{+20.4} \times 10^{20} \mathrm{~cm}^{-2}$, $\Gamma=1.10_{-0.24}^{+0.15}$, a PL model normalization of $2.9_{-0.6}^{+0.7} \times 10^{-5}$ photons $\mathrm{keV}^{-1} \mathrm{~cm}^{-2} \mathrm{~s}^{-1}$ at $1 \mathrm{keV}, k T=0.16_{-0.16}^{+0.19} \mathrm{keV}$, and $R=95.6_{-95.6}^{+927.7} d_{1 \mathrm{kpc}} \mathrm{m}$. Although the BB parameters are poorly constrained in $\phi=0.0-0.5$, we note that their best-fit values are consistent with those inferred from the phase-averaged analysis.

On the other hand, in $\phi=0.5-1$, we found that the BB component is required at $>99 \%$ confidence level. In this phase interval, the PL+BB fit yields $N_{\mathrm{H}}<4.5 \times 10^{20} \mathrm{~cm}^{-2}$, $\Gamma=1.13_{-0.26}^{+0.14}$, a PL model normalization of $7.1_{-2.1}^{+1.8} \times 10^{-6}$ photons $\mathrm{keV}^{-1} \mathrm{~cm}^{-2} \mathrm{~s}^{-1}$ at $1 \mathrm{keV}, k T=0.19_{-0.05}^{+0.04} \mathrm{keV}$, and $R=84.4_{-25.5}^{+34.1} d_{1 \mathrm{kpc}} \mathrm{m}$. The corresponding goodness-of-fit is $\chi^{2}=48.81$ for 52 dof. The BB parameters are fully consistent with those inferred in $\phi=0.0-0.5$ and the phase-averaged analysis. This suggests the thermal component provides a constant contribution in all orbital phases.

We have checked the robustness of the quoted spectral parameters by repeating all the aforementioned spectral fits with the background spectrum sampled from various sourcefree regions. Within $1 \sigma$ errors, the parameters inferred from independent fittings are consistent.

\section{FERMI/LAT GAMMA-RAY OBSERVATIONS}

Gamma-ray data were obtained, reduced, and analyzed using the Fermi Science Tools package (v9r33p0), which is available from the Fermi Science Support Center. ${ }^{11}$ Events in the reprocessed Pass 7 "Source" class were selected and the P7REP_SOURCE_V15 version of the instrumental response functions were used. To reduce contamination from the Earth's albedo, we excluded time intervals when the region-of-interest

\footnotetext{
${ }^{11}$ http://fermi.gsfc.nasa.gov/ssc/data/analysis/software/
} 
(ROI) was observed at zenith angles greater than $100^{\circ}$ or when the rocking angle of the LAT was greater than $52^{\circ}$.

For spectral analysis, we used photons between 0.1 and $300 \mathrm{GeV}$ within a $21^{\circ} \times 21^{\circ} \mathrm{ROI}$ centered at the position of 2FGL J2129.8-0428 (Nolan et al. 2012). We performed binned likelihood analyses with the gtlike tool. For source modeling, all 2FGL catalog sources (Nolan et al. 2012) within $12^{\circ}$ of the ROI center, the galactic diffuse emission (gll_iem_v05_rev1. fit), and isotropic diffuse emission (iso_source $\mathrm{v}_{\overline{05}} \mathrm{rev} 1 . \mathrm{t} \times \mathrm{t}$ ) were included. For sources more than $10^{\circ}$ away from the position of 2FGL J2129.8-0428, the spectral parameters were fixed to the catalog values.

For spectral analysis, we used Fermi/LAT data collected between 2008 August 4 and 2014 December 17. We modeled 2FGL J2129.8-0428 with a simple PL

$$
\frac{d N}{d E}=N_{0}\left(\frac{E}{E_{0}}\right)^{-\Gamma}
$$

and a power law with exponential cutoff (PLE)

$$
\frac{d N}{d E}=N_{0}\left(\frac{E}{E_{0}}\right)^{-\Gamma} \exp \left(-\frac{E}{E_{c}}\right)
$$

The fit with a simple PL gives $\Gamma=2.25 \pm 0.06$ and $F_{\gamma}=(1.57 \pm 0.20) \times 10^{-8}$ photons $\mathrm{cm}^{-2} \mathrm{~s}^{-1}$ above $100 \mathrm{MeV}$, and a test-statistic (TS; Mattox et al. 1996) value 238.2. The PL spectrum is consistent with that reported in the 2FGL catalog (Nolan et al. 2012). Although the reported 2FGL spectrum is not significantly curved, we also fit 2FGL J2129.80428 using a PLE. This gives $\Gamma=1.65 \pm 0.21$, $E_{c}=3.0 \pm 1.1 \mathrm{GeV}$, and $F_{\gamma}=(1.03 \pm 0.21) \times 10^{-8}$ photons $\mathrm{cm}^{-2} \mathrm{~s}^{-1}$ above $100 \mathrm{MeV}$, and a TS value of 258.4. The likelihood ratio test gives $2 \Delta \log ($ likelihood $) \approx 21.8$; therefore the PLE model is preferred over the PL model at a statistical significance of $\sim 4.6 \sigma$.

We also constructed a long-term light curve with threemonth bins and did not find a significant steplike flux change like that observed for PSR J1023 + 0038 in 2012 July when the latter changed from a radio MSP phase to a low-mass X-ray binary (LMXB) phase (e.g., Takata et al. 2014).

We further examine for possible gamma-ray orbital modulation using $0.1-300 \mathrm{GeV}$ photons obtained with Fermi observations over 6 yr (2008 August 4 to 2014 November 11). About 2860 counts were obtained in a circular region of $1^{\circ}$ around 2FGL J2129.8-0428. With the same phase zero as adopted in X-ray and optical investigations, the probability to randomly obtain a gamma-ray signal at least as significant as the one we obtained at the orbital period determined from optical observations, is 0.011 and 0.1 inspected through $H$ statistics $(H=11.3)$ and $\chi^{2}$ test $\left(\chi^{2}=33.1\right.$ in 24 dof $)$, respectively. So, we conclude no significant orbital modulation is detected in the gamma-ray band. The lack of orbital modulation does not support the shock origin of gamma-rays. A spectral cutoff at several $\mathrm{GeV}$ has been found from many gamma-ray MSPs (e.g., Abdo et al. 2009), and therefore the majority of the gamma-ray emission may come from the pulsar magnetosphere.

\section{DISCUSSION}

We have investigated the X-ray and UV emission from PSR J2129-0429 with XMM-Newton. Both X-ray and UV light curves are significantly modulated by the orbital period. The Xray emission is found to be non-thermal dominant with a small thermal contribution which is presumably from the neutron star surface. While the intensity of the non-thermal emission varies across the orbit, we found the thermal component is essentially constant in all orbital phases.

The X-ray modulation has a double-peaked structure with a dip in between. This is similar to the original black widow PSR B1957 + 20 (Huang et al. 2012) in which the dip appears when the secondary is between the pulsar and the Earth and its X-ray modulation can be explained by the Doppler boosting of the shocked pulsar wind that wraps the secondary star. Since we do not have any information about the radial velocity of the companion of PSR J2129-0429 from either optical spectroscopy or radio timing observations, we could not determine the geometry of its orbit with the current data. However, the phase of the X-ray dip/minimum aligns with the phase of the optical minima, suggesting the X-ray dip/minimum is at either an inferior conjunction or a superior conjunction. In this scenario, the X-ray modulation could have been caused by the Doppler boosting effect (see Huang et al. 2012 for a detailed discussion).

Optical modulation from black widow and redback MSPs is usually observed with a single broad peak since the hemisphere of the secondary star is heated by the irradiation of the strong pulsar radiations. The double peaks with 0.5 phase separation seen in Figure 2 suggests that the optical modulation of PSR J2129-0429 is not caused by the irradiation of the strong pulsar radiation. Hence, it is expected that PSR J2129-0429 belongs to the special category of redback MSPs. Table 1 summarizes the parameters of the redbacks in the Galactic field (see Romani \& Shaw 2011; Kong et al. 2012; Roberts 2013); the projected separation between two stars ( $a$ sin $i$, sixth column) is calculated from (Frank et al. 2002), $a \sin i=2.9 \times\left(M_{\mathrm{NS}} / M_{\odot}\right)^{1 / 3}(1+q)^{1 / 3}\left(P_{o} / \text { day }\right)^{2 / 3} \mathrm{~cm}$, where $M_{\mathrm{NS}}=1.4 M_{\odot}$ is the neutron star mass and $q=M_{\min } / M_{\mathrm{NS}}$ with $M_{\text {min }}$ being the inferred minimum mass (fifth column) of the secondary, and $i$ is the orbital inclination. In all the following estimations/modeling, we assume $a \sim a \sin i$. We further calculate the typical size of the Roche lobe using $r_{L}=0.462 a[q /(1+q)]^{1 / 3}$. The last column shows the ratio of the stellar luminosity without heating $\left(L_{\text {star }}\right)$ and the pulsar luminosity deposited onto the stellar surface $\left(L_{\text {heat }}\right)$, that is $\eta=L_{\text {star }} / L_{\text {heat }}$. Since we lack optical observations for most of the redbacks and the secondary star is a non-degenerate star, we infer the stellar luminosity without heating using a simple relation $L_{\text {star }} \propto M_{\text {min }}^{\beta}$ with $\beta \sim 3.5$ for a main-sequence star. For example, the effective temperature of the unheated side of the secondary is $T_{\text {eff }, 0} \sim 2900 \mathrm{~K}$ for PSR J2339-0533 (Romani \& Shaw 2011) and 5600 K for J1023+0038 (Thorstensen \& Armstrong 2005). The inferred stellar luminosities are $L_{\text {star }} \sim 4 \pi r_{L}^{2} \sigma_{\mathrm{sb}} T_{\mathrm{eff}, 0}^{4} \sim 1.8 \times 10^{31} \mathrm{erg} \mathrm{s}^{-1}$ for J2339-0533 and $\sim 5.2 \times 10^{32} \mathrm{erg} \mathrm{s}^{-1}$ for $\mathrm{J} 1023+0038$, respectively, which can be fitted by $L_{\text {star }} \propto M_{\text {min }}^{\beta}$ with an index $\beta \sim 3.5$. In the calculation, we used the parameter of PSR J2333-0533 for the normalization. The heating luminosity $L_{\text {heat }}$ is calculated from $L_{\text {heat }}=f_{\Omega} L_{\text {sd }}$, where $f_{\Omega}=\left(1-\sqrt{1-r_{L}^{2} / a^{2}}\right) / 2$. In Table 1 , we see that the stellar luminosity of the secondary of PSR J2129-0429 can significantly exceed the heating luminosity, suggesting the heating effect is negligible in the optical light curve. It is also interesting to note that the redback PSR J1723- 
Table 1

Parameters of Redbacks

\begin{tabular}{|c|c|c|c|c|c|c|c|}
\hline PSR & $\begin{array}{c}P_{s} \\
(\mathrm{~ms})\end{array}$ & $\begin{array}{c}L_{\mathrm{sd}} \\
\left(10^{34} \mathrm{erg} / \mathrm{s}\right)\end{array}$ & $\begin{array}{c}P_{o} \\
\text { (day) }\end{array}$ & $\begin{array}{l}M_{\min } \\
\left(M_{\odot}\right)\end{array}$ & $\begin{array}{c}a \sin i \\
\left(10^{11} \mathrm{~cm}\right)\end{array}$ & $\begin{array}{c}r_{L} \\
\left(10^{10} \mathrm{~cm}\right)\end{array}$ & $\eta$ \\
\hline $\mathrm{J} 1023+0038$ & 1.7 & 5 & 0.2 & 0.2 & 1.2 & 2.7 & 0.8 \\
\hline J1628-32 & 3.2 & 2 & 0.21 & 0.16 & 1.2 & 2.6 & 1.2 \\
\hline J1723-2837 & 1.9 & 5 & 0.62 & 0.4 & 2.6 & 7.2 & 6.3 \\
\hline $\mathrm{J} 1816+4510$ & 3.2 & 5 & 0.36 & 0.16 & 1.7 & 3.7 & 0.4 \\
\hline $\mathrm{J} 2215+5135$ & 2.6 & 6 & 0.18 & 0.22 & 1.1 & 2.5 & 0.9 \\
\hline J2129-0429 & 7.6 & 4 & 0.63 & 0.37 & 2.6 & 7.1 & 6.4 \\
\hline J2339-0533 & 2.3 & 2 & 0.2 & 0.075 & 1.1 & 1.9 & 0.1 \\
\hline
\end{tabular}

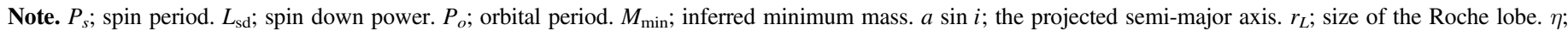
ratio of stellar luminosity without heating to heating luminosity.

2838 has orbital properties similar to those of PSR J2129-0429. PSR J1723-2838 may be another candidate that shows no heating effect in the optical light curve. Apart from the pulsar wind, the irradiation of the $\mathrm{GeV}$ gamma-rays from the pulsar is a possible heating mechanism for the secondary star in black widows/redbacks (cf. Takata et al. 2010, 2012). In such a case, the absence of heating of PSR J2129-0429 suggests the gamma-ray beam is not directed toward its companion.

Assuming that the companion of PSR J2129-0429 fills the Roche lobe, we constrain the viewing angle of the system by modeling the UV light curve. We assume the Roche lobe filling factor to be unity and the observed UV photons are produced on the Roche lobe surface (i.e., optical depth is unity on the Roche lobe surface). The Roche equipotential is given by (Frank et al. 2002)

$$
\begin{aligned}
\Phi(x, y, z)= & \frac{2}{1+q} \frac{1}{\sqrt{\left(\frac{x}{a}\right)^{2}+\left(\frac{y}{a}\right)^{2}+\left(\frac{z}{a}\right)^{2}}} \\
& +\frac{2 q}{1+q} \frac{1}{\sqrt{\left(\frac{x}{a}-1\right)^{2}+\left(\frac{y}{a}\right)^{2}+\left(\frac{z}{a}\right)^{2}}} \\
& +\frac{x}{a}\left(\frac{x}{a}-\frac{2 q}{q+1}\right)+\left(\frac{y}{a}\right)^{2},
\end{aligned}
$$

where we assume two stars orbit on the $x-y$ plane, and we used $q \sim 0.2$ of PSR J2129-0429. The Roche lobe surface is defined by the plane $\Phi(x, y, z)=\Phi\left(x_{L 1}, 0,0\right)$, where the inner Lagrangian point $x_{L 1}$ is calculated from

$$
\begin{aligned}
&- \frac{1}{1+q} \frac{1}{\left(\frac{x_{L 1}}{a}\right)^{2}}+\frac{q}{1+q} \frac{1}{\left(\frac{x_{L 1}}{a}-1\right)^{2}} \\
&+\frac{x_{L 1}}{a}-\frac{q}{1+q}=0, \quad 0<x_{L 1}<a .
\end{aligned}
$$

We divided the Roche lobe surface into many segments. For the limb darkening of each segment, we used a simple linear law, $I(\mu) \propto 1-\kappa(1-\mu)($ van Hamme 1993), where $\mu$ is the cosine of the angle between the local vector normal to the Roche lobe surface and the Earth viewing angle. We chose a typical limb darkening coefficient $\kappa=0.5$. We ignored the effects of the gravity darkening (Lucy 1967) for simplicity. Hence the observed brightness of each segment is proportional to the intensity $I(\mu)$ times the projected segment area. In the bottom panel of Figure 2, we compare the UV light curve and the model light curves for various Earth viewing angles $\xi$, where $\xi=90^{\circ}$ corresponds to the edge-on view. In Figure 2, we can see that a larger viewing angle is preferred. This can explain the presence of an extensive radio eclipse and the X-ray orbital modulation caused by the Doppler boosting effect. Since the current errors of the optical data are huge, high signal-to-noise optical observations will be required to tightly constrain the viewing geometry.

C.Y.H. is supported by the National Research Foundation of Korea through grant 2014R1A1A2058590. C.P.H. is supported by the Ministry of Science and Technology of Taiwan through the grant NSC 102-2112-M-008-020-MY3 and NSC 1012119-M-008-007-MY3. S.M.P. is supported by the BK21 plus program. A.K.H.K. and K.L.L. are supported by the Ministry of Science and Technology of Taiwan through the grant 1032628-M-007-003-MY3. P.H.T. is supported by the One Hundred Talents Program of the Sun Yat-Sen University. J.T. and K.S.C. are supported by a 2014 GRF grant of the Hong Kong Government under HKU 17300814P.

\section{REFERENCES}

Abdo, A. A., Ackermann, M., Ajello, M., et al. 2009, Sci, 325, 848 Archibald, A. M., Kaspi, V. M., Bogdanov, S., et al. 2010, ApJ, 722, 88 Bellm, E., Djorgovski, S. G., Drake, A. J., et al. 2013, BAAS, \#221, \#154.10 Bogdanov, S., Archibald, A. M., Hessels, J. W. T., et al. 2011, ApJ, 742, 97 Bogdanov, S., Grindlay, J. E., \& van den Berg, M. 2005, ApJ, 630, 1029

Bogdanov, S., Patruno, A., Archibald, A. M., et al. 2014, ApJ, 789, 40

Frank, J., King, A., \& Raine, D. J. 2002, Accretion Power in Astrophysics (Cambridge, UK: Cambridge Univ. Press)

Gentile, P. A., Roberts, M. S. E., McLaughlin, M. A., et al. 2014, ApJ, 783, 69

Hessels, J. W. T., Roberts, M. S. E., McLaughlin, M. A., et al. 2011, in AIP Conf. Proc. 1357, Radio Pulsars: An Astrophysical Key to Unlock the Secrets of the Universe, ed. M. Burgay et al. (Melville, NY: AIP), 40

Huang, R. H. H., Kong, A. K. H., Takata, J., et al. 2012, ApJ, 760, 92

Hui, C. Y. 2014, JASS, 31, 101

Hui, C. Y., Park, S. M., Hu, C. P., et al. 2015, ApJ, submitted

Hui, C. Y., Tam, P. H. T., Takata, J., et al. 2014, ApJL, 781, L21

Kong, A. K. H., Huang, R. H. H., Cheng, K. S., et al. 2012, ApJL, 747, L3

Kong, A. K. H., Jin, R., Yen, T.-C., et al. 2014, ApJL, 794, L22

Li, K. L., Kong, A. K. H., Takata, J., et al. 2014, ApJ, 797, 111

Linares, M. 2014, ApJ, 795, 72

Lucy, L. B. 1967, ZA, 65, 89

Mattox, J. R., Bertsch, D. L., Chiang, J., et al. 1996, ApJ, 461, 396

Nolan, P. L., Abdo, A. A., Ackermann, M., et al. 2012, ApJS, 199, 31

Ray, P. S., Abdo, A. A., Parent, D., et al. 2012, in Fermi Symp. Proc.-eConf. C110509 (arXiv:1205.3089) 
Roberts, M. S. E. 2013, Proc. IAU, 291, 127

Roberts, M. S. E. 2014, Proc. 5th Int. Fermi Symp. (arXiv:1502.07208)

Romani, R. W., \& Shaw, M. S. 2011, ApJL, 743, 26

Takata, J., Cheng, K. S., Taam, \& Ronald, E. 2010, ApJL, 723, L68
Takata, J., Cheng, K. S., Taam, \& Ronald, E. 2012, ApJ, 745, 100

Takata, J., Li, K. L., Leung, G. C. K., et al. 2014, ApJ, 785, 131

Thorstensen, J. R., \& Armstrong, E. 2005, AJ, 130, 759

van Hamme, W. 1993, AJ, 106, 2096 